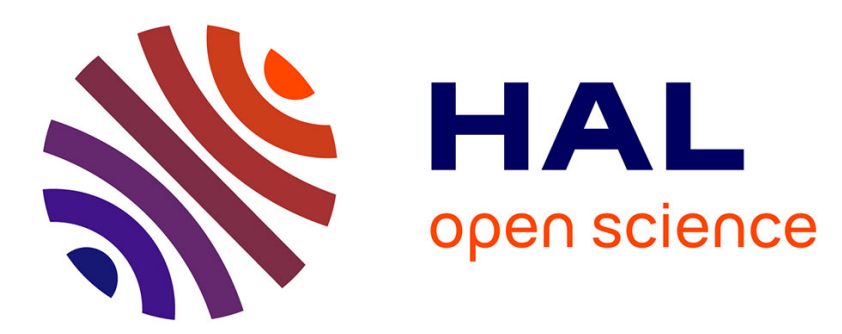

\title{
A global/local probabilistic approach for reduced-order modeling adapted to the low- and mid-frequency structural dynamics
}

Anas Batou

\section{- To cite this version:}

Anas Batou. A global/local probabilistic approach for reduced-order modeling adapted to the lowand mid-frequency structural dynamics. Computer Methods in Applied Mechanics and Engineering, 2015, 294, pp.123-140. 10.1016/j.cma.2015.06.007 . hal-01162722

HAL Id: hal-01162722

https://hal.science/hal-01162722

Submitted on 18 Jul 2015

HAL is a multi-disciplinary open access archive for the deposit and dissemination of scientific research documents, whether they are published or not. The documents may come from teaching and research institutions in France or abroad, or from public or private research centers.
L'archive ouverte pluridisciplinaire HAL, est destinée au dépôt et à la diffusion de documents scientifiques de niveau recherche, publiés ou non, émanant des établissements d'enseignement et de recherche français ou étrangers, des laboratoires publics ou privés. 


\title{
A global/local probabilistic approach for reduced-order modeling adapted to the low- and mid-frequency structural dynamics
}

\author{
Anas Batou* \\ * Université Paris-Est, Laboratoire Modélisation et Simulation Multi Echelle, MSME \\ UMR 8208 CNRS, 5 bd Descartes, 77454 Marne-la-Vallée, France, \\ E-mail: anas.batou@univ-paris-est.fr, \\ Tel.: +33160957r92, fax: +331608577999
}

\begin{abstract}
The research presented here is devoted to the construction of a probabilistic reduced-order computational model adapted to the low- and mid-frequency structural dynamics. The methodology presented here is based on a global/local separation of the space of admissible displacements by solving two separated eigenvalue problems in which the kinetic energy is modified. This paper presents a general framework for constructing the modified kinetic energy by introducing classes of kinematic reductions. The global/local separation allows the construction of a probabilistic model of uncertainties for which the fluctuations of the global displacements and the fluctuations of the local displacements can be controlled separately.
\end{abstract}

Keywords: High modal density, Low-frequency range, Mid-frequency range, Uncertainty quantification

\section{Introduction}

The dynamical analysis of complex structures in the low- and mid-frequency range often requires the construction of computational models having millions degrees of freedom (DOF) in order to compute the dynamical response with a sufficient accuracy. In automotive industry for instance, in order to take into account all the geometric and structural details, the computational models which are used today can reach tens of millions of DOFs. The dynamical analysis of structures using so large computational models directly 
yields two main problems. The first one is related to the computational cost of such a dynamical analysis. It is then necessary to reduce the size of the computational model without decreasing its accuracy in the frequency band of analysis. The second problem is related to the robustness of the computational model with respect to uncertainties. There are several sources of uncertainties: The first one concerns the existing variability within the specimens that the computational model has to represent. Indeed between two nominally identical structures, there may exist some slight differences due for instance to manufacturing tolerances, differences in the equipments (for instance different options for a car), the damages during the life cycle and so on (see $[16,10]$ for experimental frequency responses of nominally identical automotive vehicles). The second source of uncertainties concerns the computational model for which some parameters may not be perfectly known and for which there may exist some model uncertainties induced by the modeling choices related for instance to the constitutive laws, the type of elements, the modeling of the boundary conditions and so on. It is then necessary to take into account these two sources of uncertainties in order to compute the dynamical response with a good robustness.

The objective of this paper concerns the construction of a stochastic reduced-order computational model adapted to the low- and mid-frequency analysis of complex structures. For the low-frequency range, if the resonances are well-separated, the modal analysis method [23] allows a small-size reduced-order model to be constructed. In general the computed elastic modes are global and robust with respect to uncertainties. In the midfrequency range, due to the numerous local displacements of the flexible parts or embedded equipments, the modal density is higher and then the modal analysis method becomes less efficient because of the large number of modes that have to be calculated. For complex structures, which are made up of several structural scales (for instance a master stiff part and several flexible substructures), the low-frequency range and the mid-frequency overlap and sometimes structures can exhibit a high modal density even for low frequencies. A feature of the mid-frequency range concerns the high sensitivity of the response with respect to uncertainties. Several classes of methods have been proposed in the literature to construct a reduced-order model adapted to the mid-frequency range. Among these methods one can find fuzzy attachments methods $[27,33,34,36]$, Trefftz methods $[18,9,7,35,5,3,17,25]$, hybrid Finite Element/Statistical Energy methods, [28, 6, 19, 8, 20, 37, 24, 22] and methods using stochastic computational models [31, 14, 21]. 
The methodology presented in this paper belongs to the class of methodologies using a stochastic computational model. In [4, 32] a new methodology has been proposed in order to construct a reduced-order model for dynamical structures having a high modal density in the low-frequency range. This methodology consists in splitting the admissible displacement space into two subspaces: a space of global displacements and a space of local displacements. The basis of each of these spaces is constructed separately by solving an eigenvalue problem in which the kinetic energy is modified while the elastic energy is kept exact. The modified kinetic energies of theses two eigenvalue problems are obtained by introducing a projection operator than defines a kinematic reduction. In the previous papers [4, 32] this kinematic reduction consists in an averaging of the displacement in non-overlapping subdomains of the whole domain. In the present paper, a general framework is introduced in order to construct more accurate kinematic reductions. This extension of the previous researches allows to obtain (1) a global response of the structure in a larger frequency including the mid-frequency range and (2) a better global/local separation (a better filtering of the local displacements is performed for the global basis and a better filtering of the global displacements is performed for the local basis). Then a probabilistic model of uncertainties can be introduced for each scale (global and local) separately so that the global fluctuations and the local fluctuations can be controlled separately.

In Section 2, the reference boundary value problem is developed then in Section 3 the construction of the reduced-order problem is constructed and analysed for the continuous formulation. The global eigenvalue problem and the local eigenvalue problem are presented. In Section 4, the reducedorder computational model is derived using the Finite Element (FE) method. Section 5 is devoted to the construction of the probabilistic model of uncertainties for the global contributions of for the local contributions. Finally in Section 6, the methodology is illustrated on a numerical application which exhibits a high modal density in both the low- and mid-frequency range.

\section{Variational formulation of the boundary value problem}

The structure we are interested in is made up of a linear viscoelastic (with instantaneous memory) elastic medium occupying the domain $\Omega$ of $\mathbb{R}^{3}$, with boundary $\partial \Omega$. Let $\mathbf{x}$ be any point in $\Omega$. The external unit normal to $\partial \Omega$ is denoted by $\mathbf{n}$. In this paper, the dynamical response of the structure is constructed in the frequency domain, in the frequency band $B=\left[\omega_{\min }, \omega_{\max }\right]$. 
Let $\mathbf{u}(\mathbf{x}, \omega)$ be the displacement field defined on $\Omega$ with values in $\mathbb{C}^{3}$. The boundary $\partial \Omega$ is made up of two parts. On the part $\Gamma_{0}$ of the boundary $\partial \Omega$, there is a Dirichlet condition $\mathbf{u}=0$. On the part $\Gamma$ of the boundary $\partial \Omega$, a surface force field, $\mathbf{f}^{\operatorname{surf}}(\mathbf{x}, \omega)$ with values in $\mathbb{C}^{3}$, is applied. In addition, there is a volume force field, $\mathbf{f}^{\mathrm{vol}}(\mathbf{x}, \omega)$ with values in $\mathbb{C}^{3}$, applied in $\Omega$. We are interested in the linear response around a static equilibrium considered as the reference configuration defined by $\Omega$. The boundary value problem in the frequency domain is written, for all real $\omega$, as

$$
\begin{array}{cl}
-\omega^{2} \rho \mathbf{u}-\operatorname{div} \boldsymbol{\sigma}=\mathbf{f}^{\text {vol }} & \text { in } \Omega, \\
\mathbf{u}=\mathbf{0} & \text { on } \Gamma_{0}, \\
\boldsymbol{\sigma} \mathbf{n}=\mathbf{f}^{\text {surf }} & \text { on } \Gamma,
\end{array}
$$

where $\rho(\mathbf{x})$ is the positive-valued mass density, $\boldsymbol{\sigma}(\mathbf{x}, \omega)$ is the second-order stress tensor, in which $\{\operatorname{div} \boldsymbol{\sigma}(\mathbf{x}, \omega)\}_{j}=\sum_{k=1}^{3} \partial \sigma_{j k}(\mathbf{x}, \omega) / \partial x_{k}$. Let $\boldsymbol{\varepsilon}(\mathbf{x}, \omega)$ be the strain tensor defined by $\varepsilon_{j k}(\mathbf{x}, \omega)=\left(\partial u_{j}(\mathbf{x}, \omega) / \partial x_{k}+\partial u_{k}(\mathbf{x}, \omega) / \partial x_{j}\right) / 2$. The stress tensor $\boldsymbol{\sigma}(\mathbf{x}, \omega)$ is related to the strain tensor $\varepsilon(\mathbf{x}, \omega)$ by a constitutive equation which is written for a nonhomogeneous anisotropic dissipative elastic medium as $\sigma_{h \ell}(\mathbf{x}, \omega)=a_{h \ell j k}(\mathbf{x}) \varepsilon_{j k}(\mathbf{x}, \omega)+i \omega b_{h \ell j k}(\mathbf{x}) \varepsilon_{j k}(\mathbf{x}, \omega)$, in which $a_{h \ell j k}(\mathbf{x})$ and $b_{h \ell j k}(\mathbf{x})$ are the fourth-order real tensors related to the elastic and dissipative parts and which must satisfy symmetry and positiveness properties. Since $\mathbf{u}=0$ on the part $\Gamma_{0}$ of the boundary, there will be no rigid body displacements.

Let $\mathcal{H}^{\operatorname{comp}}$ be the space of all the square integrable functions defined on $\Omega$ with values in $\mathbb{C}^{3}$ equipped with inner-product $\langle\mathbf{u}, \mathbf{v}\rangle=\int_{\Omega} \mathbf{u}(\mathbf{x}) \cdot \overline{\mathbf{v}(\mathbf{x})} d \mathbf{x}$, in which $\mathbf{u} . \mathbf{v}=\sum_{i=1}^{3} u_{i} v_{i}$ is the Euclidean inner product in $\mathbb{R}^{3}$. Let $\mathcal{V}^{\text {comp }}$ be the admissible displacements space (subspace of $\mathcal{H}^{\text {comp }}$ ). The variational formulation of the boundary value problem defined by Eq. (1) consists in finding, for all $\omega$ in $B$, the function $\mathbf{u}(\omega)$ with values in $\mathcal{V}^{\text {comp }}$ such that for all $\mathbf{v}$ in $\mathcal{V}^{\text {comp }}$,

$$
-\omega^{2} m(\mathbf{u}, \mathbf{v})+i \omega d(\mathbf{u}, \mathbf{v})+k(\mathbf{u}, \mathbf{v})=g(\mathbf{v} ; \omega) .
$$

In Eq. (2), the mass sesquilinear form $m$ in defined on $\mathcal{H}^{\text {comp }} \times \mathcal{H}^{\text {comp }}$ by $m(\mathbf{u}, \mathbf{v})=\int_{\Omega} \rho \mathbf{u} \cdot \overline{\mathbf{v}} d \mathbf{x}$. The damping sesquilinear form $d$ is defined on $\mathcal{V}^{c o m p} \times$ $\mathcal{V}^{\text {comp }}$ by $d(\mathbf{u}, \mathbf{v})=\int_{\Omega} b_{i j k h}(\mathbf{x}) \epsilon_{k h}(\mathbf{u}) \epsilon_{i j}(\overline{\mathbf{v}}) d \mathbf{x}$. The stiffness sesquilinear form $k$ is defined on $\mathcal{V}^{c o m p} \times \mathcal{V}^{c o m p}$ by $k(\mathbf{u}, \mathbf{v})=\int_{\Omega} a_{i j k h}(\mathbf{x}) \epsilon_{k h}(\mathbf{u}) \epsilon_{i j}(\overline{\mathbf{v}}) d \mathbf{x}$. 
The antilinear form $g$ of the external forces is defined on $\mathcal{V}^{\text {comp }}$ by $g(\mathbf{v} ; \omega)=$ $\int_{\Gamma} f_{\text {surf }, i}(\mathbf{x}, \omega) \overline{v_{i}}(\mathbf{x}) d s+\int_{\Omega} f_{\text {vol }, i}(\mathbf{x}, \omega) \overline{v_{i}}(\mathbf{x}) d \mathbf{x}$.

\section{Construction of the reduced-order model.}

\subsection{Physical insights and summary of the methodology.}

As explained in the introduction, we are interested in complex dynamical structures which are made up of several structural scales (for instance a master stiff part and several flexible substructures) and then for which the low-frequency range and the mid-frequency overlap. Then the well-separated global modes classically encountered in LF band and the numerous local modes classically encountered in the MF and $\mathrm{HF}$ bands are intertwined and mixed the ones with the others. In general, the global displacements of a structure are robust with respect to uncertainties (except for uncertainties related to boundary conditions). On the contrary the local displacements are more sensitive with respect to uncertainties related to the numerous local details of the structure which are generally not perfectly modeled. Therefore, it is interesting to separate the global displacement from the local displacements in order to model the uncertainties related to global displacements independently from the uncertainties related to the local displacements. For the complex structures we are interested in, the elastic modes are combinations of global displacements and local displacements and therefore a separation is not possible.

The objective here is to construct a basis for the global displacements and a basis for the local displacements separately. The key idea for this construction consists in modifying dynamically the mass distribution of the structure in order to either (1) lock the local displacements and then keep only global displacements or (2) lock the global displacements and then keep only local displacements. We then obtain two new computational models corresponding to these two modifications of the mass distribution and for which the stiffness properties are kept unchanged with respect to the original structure. Then the global basis and the local basis correspond to the elastic modes associated with each of these two new computational models. It is important to keep the stiffness of the structure exact so that the global basis and the local basis return the boundary conditions and the static deformations of the original structure and then the union these two basis provides a good projection basis for the original structure. This locking methodology is directly inspired by the lumping mass method [13] which consists in concentrating the mass 
at given master points in order to extract a global basis. Nevertheless, the methodology we propose is more general and allows the global basis to be completed by constructing separately a local basis.

The dynamical modification of the mass distribution is performed by reducing the kinematics of the displacement associated with the kinetic energy (while the kinematics associated with the elastic energy is not modified). For instance, if for a deformable structure the kinematics of the kinetic energy is reduced to homogeneous displacements for the three directions, then the total mass of the structure is equivalent a point mass associated with three DOFs corresponding to three spacial averagings of the displacement along the three directions (see first particular case below). As a consequence the structure associated with this new kinetic energy will only exhibit three global elastic modes. A complementary kinematic reduction can be associated with each kinematic reduction. Then the kinematic reduction and the complementary kinetic reduction allows to split the kinetic energy into (1) a reduced kinetic which is used for the construction of the global basis and (2) a complementary kinetic energy which is used for the construction of the local basis.

From a mathematical point of view, the kinematic reduction and the complementary kinematic reduction correspond to a projection operator and its complementary projection operator respectively. This framework allows :

- to construct kinematic reductions that make the reduced kinetic energy as closed as possible to the original kinetic energy.

- to split properly the kinetic energy into a reduced kinetic and a complementary kinetic energy.

- to obtain a global basis and a local basis for which the union forms a basis of the admissible displacements space.

In this Section, we first present the construction of the kinematic reductions using projection operators. For a given class of kinematic reductions, the optimal one is constructed as the one that minimizes the kinetic energy associated with the errors introduced by the kinetic reduction. Three particular interesting cases are developed. Then the reduced kinetic energy and the complementary reduced kinetic energy are constructed by projecting the initial kinetic energy using the kinematic reduction and the complementary kinetic reduction. Finally, a global eigenvalue problem and a local eigenvalue problem can be constructed by replacing the original kinetic energy by the reduced kinetic energy and the complementary kinetic energy respectively (the elastic energy is kept exact). These two eigenvalue problems are solved 
separately and their solutions provide a global basis and a local basis which are independent and for which the union forms a basis of the admissible displacements space.

\subsection{Kinematic reduction of the kinetic energy.}

\subsubsection{General methodology}

Let $\mathcal{H}$ be the subspace of $\mathcal{H}^{\text {comp }}$ of real square integrable functions and $\mathcal{V}$ be the subspace of $\mathcal{V}^{c o m p}$ made of real functions. The kinematic reduction is performed using the projection operator $\mathbf{u} \mapsto \mathbf{h}^{r}(\mathbf{u})$ defined on $\mathcal{H}$ with values in a $n_{r}$-finite dimension subspace $\mathcal{H}^{r}$ of $\mathcal{H}$. Then the complementary projection operator $\mathbf{u} \mapsto \mathbf{h}^{c}(\mathbf{u})=\mathbf{u}-\mathbf{h}^{r}(\mathbf{u})$ defined on $\mathcal{H}$ with values in a subspace $\mathcal{H}^{c}$ of $\mathcal{H}$ can also be constructed. Let $\mathbf{u}^{r}=\mathbf{h}^{r}(\mathbf{u})$ and $\mathbf{u}^{c}=\mathbf{h}^{c}(\mathbf{u})$. By construction we have $\mathcal{H}=\mathcal{H}^{r} \oplus \mathcal{H}^{c}$.

We now have to construct the projection operator $\mathbf{h}^{r}$. Let $m(\mathbf{u}, \mathbf{v})=$ $\int_{\mathbb{R}^{3}} \rho(\mathbf{x}) \mathbf{u}(\mathbf{x}) \cdot \mathbf{v}(\mathbf{x}) \mathrm{d} \mathbf{x}$ be the mass symmetric positive-definite bilinear form defined on $\mathcal{H} \times \mathcal{H}$. Let endow the space $\mathcal{H}$ with the inner-product $<\mathbf{u}, \mathbf{v}>_{m}=$ $m(\mathbf{u}, \mathbf{v})$ with associate norm $\|\mathbf{u}\|_{m}=m(\mathbf{u}, \mathbf{u})^{1 / 2}$. Then the projection $\mathbf{u}^{r}(\mathbf{x})$ of function $\mathbf{u}(\mathbf{x})$ is constructed as the function minimizing the distance $\|$ $\mathbf{u}-\mathbf{u}^{r} \|_{m}$ in which $\mathbf{u}^{r}$ belongs to $\mathcal{H}^{r}$. Physically, this construction consists in minimizing the kinetic energy of the error associated to the projection. Let $\left\{\mathbf{g}_{1}^{r}(\mathbf{x}), \ldots, \mathbf{g}_{n_{r}}^{r}(\mathbf{x})\right\}$ be a basis of $\mathcal{H}^{r}$ and let $[G]=\left[\mathbf{g}_{1}^{r} \ldots \mathbf{g}_{n_{r}}^{r}\right]$. Then any function $\mathbf{u}^{r}$ of $\mathcal{H}^{r}$ can be written as

$$
\mathbf{u}^{r}(\mathbf{x})=[G(\mathbf{x})] \boldsymbol{\delta}(\mathbf{u})
$$

where the vector $\boldsymbol{\delta}(\mathbf{u})$ contains the coordinates of $\mathbf{u}^{r}$ in the basis $\left\{\mathbf{g}_{1}^{r}(\mathbf{x}), \ldots\right.$, $\left.\mathbf{g}_{n_{r}}^{r}(\mathbf{x})\right\}$ which correspond to dynamical degrees of freedom related to the kinematic reduction. We then have to calculate the optimal vector $\boldsymbol{\delta}^{\text {opt }}(\mathbf{u})$ which is solution of the minimization problem

$$
\boldsymbol{\delta}^{\mathrm{opt}}(\mathbf{u})=\underset{\boldsymbol{\delta} \in \mathbb{R}^{n_{r}}}{\arg \min _{1}}\|\mathbf{u}-[G] \boldsymbol{\delta}\|_{m}^{2} .
$$

It can easily be shown that the solution of the mean-square minimization problem (4) is given by

$$
\boldsymbol{\delta}^{\mathrm{opt}}(\mathbf{u})=[\mathcal{M}]^{-1} \mathbf{c}(\mathbf{u})
$$


where the matrix $[\mathcal{M}]$ and the vector $\mathbf{c}(\mathbf{u})$ are defined by

$$
[\mathcal{M}]=\left[\int_{\mathbb{R}^{3}} \rho(\mathbf{x})[G(\mathbf{x})]^{T}[G(\mathbf{x})] \mathrm{d} \mathbf{x}\right] \quad \text { et } \quad \mathbf{c}(\mathbf{u})=\int_{\mathbb{R}^{3}} \rho(\mathbf{x})[G(\mathbf{x})]^{T} \mathbf{u}(\mathbf{x}) \mathrm{d} \mathbf{x} .
$$

Then the projection $\mathbf{u}^{r}$ is written as

$$
\mathbf{u}^{r}=\mathbf{h}^{r}(\mathbf{u})=[G][\mathcal{M}]^{-1} \mathbf{c}(\mathbf{u}),
$$

and the complementary projection $\mathbf{u}^{c}$ is written as

$$
\mathbf{u}^{c}=\mathbf{h}^{c}(\mathbf{u})=\mathbf{u}-[G][\mathcal{M}]^{-1} \mathbf{c}(\mathbf{u}) .
$$

The orthogonal projection theorem [2] states that (1) the solutions $\mathbf{u}^{r}$ and $\mathbf{u}^{c}$ given by Eqs.(7) and (8) exist and are unique and (2) for all $\mathbf{v}^{r}$ in $\mathcal{H}^{r}$

$$
m\left(\mathbf{u}^{c}, \mathbf{v}^{r}\right)=0
$$

and thus

$$
m\left(\mathbf{u}^{c}, \mathbf{u}^{r}\right)=0 .
$$

The reduced mass bilinear form $m^{r}(\mathbf{u}, \mathbf{v})$ is defined by

$$
m^{r}(\mathbf{u}, \mathbf{v})=m\left(\mathbf{u}^{r}, \mathbf{v}^{r}\right),
$$

Then using the decomposition $\mathbf{u}=\mathbf{u}^{r}+\mathbf{u}^{c}$ and Eq.(10), we have

$$
m^{r}(\mathbf{u}, \mathbf{v})=m\left(\mathbf{u}, \mathbf{v}^{r}\right)=m\left(\mathbf{u}^{r}, \mathbf{v}\right) .
$$

This reduced mass bilinear form can be expressed as

$$
m^{r}(\mathbf{u}, \mathbf{v})=\boldsymbol{\delta}^{\mathrm{opt}}(\mathbf{u})^{T}[\mathcal{M}] \boldsymbol{\delta}^{\mathrm{opt}}(\mathbf{v})=\mathbf{c}(\mathbf{u})^{T}[\mathcal{M}]^{-1} \mathbf{c}(\mathbf{v}) .
$$

Similarly, the complementary bilinear form $m^{c}(\mathbf{u}, \mathbf{v})$ is defined by

$$
m^{c}(\mathbf{u}, \mathbf{v})=m\left(\mathbf{u}^{c}, \mathbf{v}^{c}\right)=m\left(\mathbf{u}, \mathbf{v}^{c}\right)=m\left(\mathbf{u}^{c}, \mathbf{v}\right) .
$$

Using Eq.(10), we then have

$$
m^{c}(\mathbf{u}, \mathbf{v})=m(\mathbf{u}, \mathbf{v})-m^{r}(\mathbf{u}, \mathbf{v}) .
$$


This relation shows that the initial kinetic energy (represented by the mass bilinear form) is split into a reduced kinetic energy (represented by the reduced mass bilinear form) and a complementary kinetic energy (represented by the complementary mass bilinear form).

\subsubsection{Particular cases}

The matrix $[\mathcal{M}]$ and the vector $\mathbf{c}(\mathbf{u})$ depend on the choice for the subspace $\mathcal{H}^{r}$. The particular case that are developed below correspond to approximations which are based on the decomposition of the domain $\Omega$ into $n_{J}$ non-overlapping subdomains $\Omega_{j}$. The size of these subdomains controls the size of the smallest spatial wavelengths for the displacements that will be kept in the global basis. For these particular cases, only the projection operators are derived. The complementary projection operators are deduced easily using Eq. (8).

\section{Particular case 1: Uniform displacement in each subdomain.}

For this first case, the displacement $\mathbf{u}$ is approximated by a displacement $\mathbf{u}^{r}$ which is uniform in each subdomain $\Omega_{j}$ :

$$
\mathbf{u}^{r}(\mathbf{x})=\sum_{j=1}^{n_{J}} \mathbb{1}_{\Omega_{j}}(\mathbf{x}) \mathbf{u}_{0, j}(\mathbf{u})
$$

In this case $\boldsymbol{\delta}(\mathbf{u})=\left(\mathbf{u}_{0,1}(\mathbf{u})^{T}, \ldots, \mathbf{u}_{0, n_{J}}(\mathbf{u})^{T}\right)^{T}$ and $n_{r}=3 n_{J}$. We then have $[G(\mathbf{x})]=\left[\mathbb{1}_{\Omega_{1}}(\mathbf{x})\left[I_{3}\right], \ldots, \mathbb{1}_{\Omega_{n_{J}}}(\mathbf{x})\left[I_{3}\right]\right]$ and it can be shown that

$$
[\mathcal{M}]=\left[\begin{array}{ccc}
m_{1}\left[I_{3}\right] & \cdots & 0 \\
\vdots & \ddots & \vdots \\
0 & \cdots & m_{n_{J}}\left[I_{3}\right]
\end{array}\right], \boldsymbol{\delta}^{\mathrm{opt}}(\mathbf{u})=\left[\begin{array}{c}
\frac{1}{m_{1}} \int_{\Omega_{1}} \rho\left(\mathbf{x}^{\prime}\right) \mathbf{u}\left(\mathbf{x}^{\prime}\right) d \mathbf{x}^{\prime} \\
\vdots \\
\frac{1}{m_{n_{J}}} \int_{\Omega_{n_{J}}} \rho\left(\mathbf{x}^{\prime}\right) \mathbf{u}\left(\mathbf{x}^{\prime}\right) d \mathbf{x}^{\prime}
\end{array}\right],
$$

where $m_{j}=\int_{\Omega_{j}} \rho(\mathbf{x}) \mathrm{d} \mathbf{x}$ is the mass of subdomain $\Omega_{j}$. In Eq. (17), the matrix $[\mathcal{M}]$ corresponds to the mass matrix of $n_{J}$ uncoupled point masses $m_{1}, \ldots, m_{n_{J}}$ and the dynamical degrees of freedom $\boldsymbol{\delta}^{\mathrm{opt}}(\mathbf{u})$ correspond to averagings, in each subdomain, of the displacement $\mathbf{u}$ with respect to the mass density $\rho$. Finally, the projection $\mathbf{u}^{r}$ of the displacement $\mathbf{u}$ is written as

$$
\mathbf{u}^{r}(\mathbf{x})=\sum_{j=1}^{n_{J}} \mathbb{1}_{\Omega_{j}}(\mathbf{x}) \frac{1}{m_{j}} \int_{\Omega_{j}} \rho\left(\mathbf{x}^{\prime}\right) \mathbf{u}\left(\mathbf{x}^{\prime}\right) d \mathbf{x}^{\prime},
$$


and the corresponding reduced mass bilinear form is written as

$$
m^{r}(\mathbf{u}, \mathbf{v})=\sum_{j=1}^{n_{J}} m_{j}\left\|\frac{1}{m_{j}} \int_{\Omega_{j}} \rho\left(\mathbf{x}^{\prime}\right) \mathbf{u}\left(\mathbf{x}^{\prime}\right) d \mathbf{x}^{\prime}\right\|^{2} .
$$

The projection defined by Eq. (18) for this first particular case corresponds to the projection operator that has been presented in the previous papers $[4,32]$ and can be interpreted as an averaging of the displacement $\mathbf{u}$, in each subdomain. It should be noted that the reduction of the kinetic energy defined by Eq. (19) does not correspond to a classical mass lumping for which the masses of the subdomains are concentrated at given master points. Here the masses of the subdomains are distributed to dynamical degrees of freedom which corresponds to averagings of the displacement.

\section{Particular case 2: Rigid body displacement in each subdomain.}

The previous particular case can be enriched by taking into account the rotations of the subdomains which can become large in the mid-frequency range. For this second particular case, these rotations are taken into account by approximating the displacement $\mathbf{u}$ by a (small) rigid body displacement in each subdomain $\Omega_{j}$ :

$$
\mathbf{u}^{r}(\mathbf{x})=\sum_{j=1}^{n_{J}} \mathbb{1}_{\Omega_{j}}(\mathbf{x})\left\{\mathbf{u}_{0, j}(\mathbf{u})+\mathbf{u}_{1, j}(\mathbf{u}) \times\left(\mathbf{x}-\mathbf{r}_{j}\right)\right\}
$$

in which $(\cdot \times \cdot)$ denotes the cross-product and where $\mathbf{r}_{i}=\left(1 / m_{j}\right) \int_{\Omega_{j}} \mathbf{x} \rho(\mathbf{x}) \mathrm{d} \mathbf{x}$ is the center of mass of subdomain $\Omega_{j}$. In this case, $\boldsymbol{\delta}(\mathbf{u})=\left(\mathbf{u}_{0,1}(\mathbf{u})^{T}, \mathbf{u}_{1,1}(\mathbf{u})^{T}, \ldots\right.$, $\left.\mathbf{u}_{0, n_{J}}(\mathbf{u})^{T}, \mathbf{u}_{1, n_{J}}(\mathbf{u})^{T}\right)^{T}$ and $n_{r}=6 n_{J}$. The reduced mass matrix is then defined by

$$
[\mathcal{M}]=\left[\begin{array}{ccccc}
m_{1}\left[I_{3}\right] & 0 & \cdots & 0 & 0 \\
0 & {\left[J_{1}\right]} & \cdots & 0 & 0 \\
\vdots & \vdots & \ddots & \vdots & \vdots \\
0 & 0 & \cdots & m_{n_{J}}\left[I_{3}\right] & 0 \\
0 & 0 & \cdots & 0 & {\left[J_{n_{J}}\right]}
\end{array}\right]
$$

where $\left[J_{j}\right]$ is the inertia matrix of subdomain $\Omega_{j}$ such that

$$
\left[J_{j}\right]=\operatorname{tr}\left(\left[Z_{j}\right]\right)\left[I_{3}\right]-\left[J_{j}\right]
$$


in which $\left[Z_{j}\right]$ is the $(3 \times 3)$ matrix related to the Euler tensor and is defined by

$$
\left[Z_{j}\right]=\int_{\Omega_{j}}\left(\mathbf{x}-\mathbf{r}_{i}\right)\left(\mathbf{x}-\mathbf{r}_{i}\right)^{T} \rho(\mathbf{x}) d \mathbf{x}
$$

In Eq. (21), the reduced matrix [ $\mathcal{M}]$ corresponds to the mass matrix of $n_{J}$ uncoupled rigid bodies. Finally, the projection $\mathbf{u}^{r}$ of the displacement $\mathbf{u}$ is written as

$$
\begin{aligned}
\mathbf{u}^{r}(\mathbf{x})=\sum_{j=1}^{n_{J}} \mathbb{1}_{\Omega_{j}}(\mathbf{x})\left\{\frac{1}{m_{j}} \int_{\Omega_{j}} \rho\left(\mathbf{x}^{\prime}\right) \mathbf{u}\left(\mathbf{x}^{\prime}\right) d \mathbf{x}^{\prime}\right. \\
\left.+\left[J_{j}\right]^{-1}\left(\int_{\Omega_{j}} \rho\left(\mathbf{x}^{\prime}\right)\left(\mathbf{x}^{\prime}-\mathbf{r}_{j}\right) \times \mathbf{u}\left(\mathbf{x}^{\prime}\right) d \mathbf{x}^{\prime}\right) \times\left(\mathbf{x}-\mathbf{r}_{j}\right)\right\}
\end{aligned}
$$

It should be noted that the displacement $\mathbf{u}$ could be approximated by a large rigid body displacement in each subdomain $\Omega_{j}$ :

$$
\mathbf{u}^{r}(\mathbf{x})=\sum_{j=1}^{n_{J}} \mathbb{1}_{\Omega_{j}}(\mathbf{x})\left\{\mathbf{u}_{0, j}(\mathbf{u})+\left[\operatorname{Rot}_{j}(\mathbf{u})\right]\left(\mathbf{x}-\mathbf{r}_{j}\right)\right\}
$$

where $\left[\operatorname{Rot}_{j}(\mathbf{u})\right]$ would be a rotation matrix. Unfortunately, using such an approximation, the subspace $\mathcal{H}^{r}$ would not be a subspace of $\mathcal{H}$ (the linear combination of two rotation matrix is not, in general, a rotation matrix). Nevertheless, such an approximation, which corresponds to the construction of Tisserand axes (see [11]) can be constructed under some hypotheses [11, 12] which ensure the existence and the uniqueness of the solution of the minimization problem (4).

\section{Particular case 3: Linear displacement in each subdomain.}

This third particular case corresponds to a linear approximation of the displacement $\mathbf{u}$ is each subdomain $\Omega_{j}$ :

$$
\mathbf{u}^{r}(\mathbf{x})=\sum_{j=1}^{n_{J}} \mathbb{1}_{\Omega_{j}}(\mathbf{x})\left\{\mathbf{u}_{0, j}(\mathbf{u})+[T(\mathbf{u})]\left(\mathbf{x}-\mathbf{r}_{j}\right)\right\},
$$

where $[T(\mathbf{u})]$ is a $(3 \times 3)$ real matrix. Thus this third case corresponds to a generalization of the previous case (for which the slope matrices were 
restricted to $(3 \times 3)$ real antisymmetric matrices). In this case, it can be shown that the projection $\mathbf{u}^{r}$ is written as

$$
\begin{aligned}
\mathbf{u}^{r}(\mathbf{x})=\sum_{j=1}^{n_{J}} \mathbb{1}_{\Omega_{j}}(\mathbf{x})\{ & \frac{1}{m_{j}} \int_{\Omega_{j}} \rho\left(\mathbf{x}^{\prime}\right) \mathbf{u}\left(\mathbf{x}^{\prime}\right) d \mathbf{x}^{\prime} \\
& \left.+\left(\int_{\Omega_{j}} \rho\left(\mathbf{x}^{\prime}\right) \mathbf{u}\left(\mathbf{x}^{\prime}\right)\left(\mathbf{x}^{\prime}-\mathbf{r}_{j}\right)^{T} d \mathbf{x}^{\prime}\right)\left[Z_{j}\right]^{-1}\left(\mathbf{x}-\mathbf{r}_{j}\right)\right\},
\end{aligned}
$$

This third particular case could be generalized to higher-order polynomial approximations in each subdomain $\Omega_{j}$.

\subsection{Global eigenvalue problem and local eigenvalue problem.}

The global basis and the local basis are constructed by solving two separated eigenvalue problem for which the kinetic energy (represented by the mass bilinear form) is modified while the elastic energy (represented by the stiffness bilinear form) is kept exact:

(1) The global basis is constituted of the global eigenmodes $\varphi^{g}$ in $\mathcal{V}$ associated with the global eigenvalues $\lambda^{g}$ verifying for all $v$ in $\mathcal{V}$ the following global eigenvalues problem

$$
-\lambda^{g} m^{r}\left(\varphi^{g}, v\right)+k\left(\varphi^{g}, v\right)=0 .
$$

By replacing the kinetic energy by the reduced kinetic energy (which is chosen sufficiently rough) the mass is distributed to a small number of dynamical degrees of freedom representing the global displacements of the structure. Therefore the local displacements become massless and only global displacements are kept in the global basis.

(2) The local basis is constituted of the local eigenmodes $\varphi^{\ell}$ in $\mathcal{V}$ associated with the local eigenvalues $\lambda^{\ell}$ verifying for all $v$ in $\mathcal{V}$ the following local eigenvalues problem

$$
-\lambda^{\ell} m^{c}\left(\varphi^{\ell}, v\right)+k\left(\varphi^{\ell}, v\right)=0 .
$$

By replacing the kinetic energy by the complementary reduced kinetic energy the mass is distributed to local dynamical degrees of freedom. Therefore the global displacements become massless and only local displacements are kept in the local basis. 
The properties of these two eigenvalue problems have been studied in [32] for the particular case 1 presented in Section 3.2.2 (uniform displacement in each subdomain) only. It was proven that the global eigenvalue problem admits $n_{r}$ finite solutions $0<\lambda_{1}^{g} \leq \ldots \leq \lambda_{n_{r}}^{g}$ associated with the family of global eigenmodes $\left\{\varphi_{1}^{g}, \ldots, \varphi_{n_{r}}^{g}\right\}$ and that the local eigenvalue problem admits an infinite increasing sequence of solutions $0<\lambda_{1}^{\ell} \leq \lambda_{2}^{\ell} \leq \ldots$, associated with the family of local eigenmodes $\left\{\varphi_{1}^{\ell}, \varphi_{2}^{\ell}, \ldots\right\}$. It was also proven in [32] that the family $\left\{\varphi_{1}^{g}, \ldots, \varphi_{n_{r}}^{g}, \varphi_{1}^{\ell}, \varphi_{2}^{\ell}, \ldots\right\}$ containing both the global eigenmodes and the local eigenmodes is a basis of the space $\mathcal{V}$ and then any function $u$ in $\mathcal{V}$ can written as

$$
u=\sum_{\alpha=1}^{n_{r}} \varphi_{\alpha}^{g} q_{\alpha}^{g}+\sum_{\alpha^{\prime}=1}^{+\infty} \varphi_{\alpha^{\prime}}^{\ell} q_{\alpha^{\prime}}^{l} .
$$

In [32], the proofs concerning these properties are based on the property $\mathcal{H}=\mathcal{H}^{r} \oplus \mathcal{H}^{c}$. In the present paper, this property is verified by construction and then the properties of the eigenvalue problems listed hereinbefore are still verified.

A reduced-order model can then be constructed by truncation of the global and local bases:

$$
u \simeq \sum_{\alpha=1}^{n_{g}} \varphi_{\alpha}^{g} q_{\alpha}^{g}+\sum_{\alpha^{\prime}=1}^{n_{\ell}} \varphi_{\alpha^{\prime}}^{\ell} q_{\alpha^{\prime}}^{\ell},
$$

in which $n_{g} \leq n_{r}$ is the number of global eigenmodes which are kept in the global reduced-order basis and $n_{\ell}$ is the number of local eigenmodes which are kept in local reduced-order basis.

\section{Reduced-order computational model.}

\subsection{Full-order computational model.}

The computational model is constructed using the FE method applied to the variational formulation defined by Eq. (2). Let $m$ be the number of DOFs. Then, for all $\omega \in B$, the vector $\mathbb{U}(\omega)$ in $\mathbb{R}^{m}$ is the solution of the following matrix equation

$$
\left(-\omega^{2}[\mathbb{M}]+i \omega[\mathbb{D}]+[\mathbb{K}]\right) \mathbb{U}(\omega)=\mathbb{F}(\omega),
$$


in which $[\mathbb{M}],[\mathbb{D}]$ and $[\mathbb{K}]$ are the $(m \times m)$ positive-definite mass, damping and stiffness matrices corresponding to the FE discretization of the mass, damping and stiffness sesquilinear forms $(m, d$ and $k)$ and where $\mathbb{F}(\omega)$ is the vector of the external forces corresponding to the FE discretization of the antilinear form $g$ of the external forces.

\subsection{Global and local reduced-order bases.}

For the FE discretization, the kinetic energy is represented by the mass matrix $[\mathbb{M}]$. We then have to reduce the kinematic for the mass matrix in order to construct a reduced mass matrix $\left[\mathbb{M}^{r}\right]$ and a complementary matrix $\left[\mathbb{M}^{c}\right]$. There are three strategies in order to construct these two modified mass matrices:

(1) The first one consists in directly constructing a FE discretization of the reduced mass bilinear form $m^{r}$ and the complementary mass bilinear form $m^{c}$ defined by Eqs. (11) and (14).

(2) The second one consists in constructing a FE discretization of the projection operators $\mathbf{h}^{r}$ and $\mathbf{h}^{c}$ defined by Eqs. (7) and (8) yielding the $(m \times m)$ projection matrices $\left[H^{r}\right]$ and $\left[H^{c}\right]$ respectively. Then the reduced and complementary matrices are constructed such that $\left[\mathbb{M}^{r}\right]=\left[H^{r}\right]^{T}[\mathbb{M}]\left[H^{r}\right]$ and $\left[\mathbb{M}^{c}\right]=\left[H^{c}\right]^{T}[\mathbb{M}]\left[H^{c}\right]$.

(3) The third one, which is the one adopted here, consists in directly constructing a projection operator $\mathbb{U} \mapsto \mathbf{h}^{r}(\mathbb{U})$ with values in a $n_{r}$-dimension subspace $\mathcal{Y}^{r}$ of $\mathbb{R}^{m}$ and a complementary projection operator $\mathbb{U} \mapsto \mathbf{h}^{c}(\mathbb{U})=$ $\mathbb{U}-\mathbf{h}^{r}(\mathbb{U})$ with values in a $n_{c}$-dimension subspace $\mathcal{Y}^{c}$ of $\mathbb{R}^{m}$ such that $m=n_{r}+n_{c}$ and $\mathbb{R}^{m}=\mathcal{Y}^{r} \oplus \mathcal{Y}^{c}$. Then by equipping $\mathbb{R}^{m}$ with the innerproduct $<\mathbb{U}, \mathbb{V}>_{\mathbb{M}}=\mathbb{U}^{T}[\mathbb{M}] \mathbb{V}$ with associated norm $\|\mathbb{U}\|_{\mathbb{M}}=<\mathbb{U}, \mathbb{U}>_{\mathbb{M}}^{1 / 2}$ and by introducing a basis $\left\{\mathbf{g}_{1}^{r}, \ldots, \mathbf{g}_{n_{r}}^{r}\right\}$ of $\mathcal{Y}^{r}$, the projection $\mathbb{U}^{r}$ of vector $\mathbb{U}$ is constructed by minimizing the error $\|\mathbb{U}-[G] \delta\|_{\mathbb{M}}^{2}$, in which $[G]=\left[\mathbf{g}_{1}^{r}, \ldots, \mathbf{g}_{n_{r}}^{r}\right]$ and where $\boldsymbol{\delta}$ is the vector of the coordinates of $\mathbb{U}^{r}$ in the basis $\left\{\mathbf{g}_{1}^{r}, \ldots, \mathbf{g}_{n_{r}}^{r}\right\}$. It can then be shown that $\mathbb{U}^{r}=\left[H^{r}\right] \mathbb{U}$ in which $\left[H^{r}\right]=[G]\left([G]^{T}[\mathbb{M}][G]\right)^{-1}[G]^{T}[\mathbb{M}]$ is a $(m \times m) n_{r}$-rank matrix and $\mathbb{U}^{c}=$ $\left[H^{c}\right] \mathbb{U}$ in which $\left[H^{c}\right]=\left[I_{m}\right]-\left[H^{r}\right]$ is a $(m \times m) n_{c}$-rank matrix. This third strategy is easier to implement than the two previous one. Furthermore it allows the orthogonality property

$$
\left(\mathbb{U}^{c}\right)^{T}[\mathbb{M}] \mathbb{U}^{r}=0
$$

to be verified exactly. Then, similarly to the second strategy, the reduced 
and complementary mass matrices are constructed such that

$$
\left[\mathbb{M}^{r}\right]=\left[H^{r}\right]^{T}[\mathbb{M}]\left[H^{r}\right]=[\mathbb{M}]\left[H^{r}\right]=\left[H^{r}\right]^{T}[\mathbb{M}]
$$

and

$$
\left[\mathbb{M}^{c}\right]=\left[H^{c}\right]^{T}[\mathbb{M}]\left[H^{c}\right]=[\mathbb{M}]\left[H^{c}\right]=\left[H^{c}\right]^{T}[\mathbb{M}]
$$

The rank of $\left[\mathbb{M}^{r}\right]$ is $n_{r}$ and the rank of $\left[\mathbb{M}^{c}\right]$ is $n_{c}$.

The global eigenvectors $\phi^{g} \in \mathbb{R}^{m}$ which form the global basis are solutions the following global generalized eigenvalue problem

$$
[\mathbb{K}] \phi^{g}=\lambda^{g}\left[\mathbb{M}^{r}\right] \phi^{g} .
$$

This problems admits a family of $n_{r}$ positive finite eigenvalues $0<\lambda_{1}^{g} \leq$ $\ldots \leq \lambda_{n_{r}}^{g}$, associated with the family of global eigenvectors $\left\{\phi_{1}^{g}, \ldots, \phi_{n_{r}}^{g}\right\}$.

The local eigenvectors $\phi^{\ell} \in \mathbb{R}^{m}$ which form the local basis are solutions the following local generalized eigenvalue problem

$$
[\mathbb{K}] \phi^{\ell}=\lambda^{\ell}\left[\mathbb{M}^{c}\right] \phi^{\ell} .
$$

This problems admits a family of $n_{c}$ positive finite eigenvalues $0<\lambda_{1}^{\ell} \leq$ $\ldots \leq \lambda_{n_{c}}^{\ell}$, associated with the family of global eigenvectors $\left\{\phi_{1}^{\ell}, \ldots, \phi_{n_{c}}^{\ell}\right\}$. It should be noted that by using a subspace iteration algorithm to solve these two eigenvalue problems, the full matrices $\left[\mathbb{M}^{r}\right]$ et $\left[\mathbb{M}^{c}\right]$ do not need to be assembled.

It is shown in Appendix A that the global basis and the local basis are linearly independent. Then, since $m=n_{r}+n_{c}$, the union of these two bases is a basis of $\mathbb{R}^{m}$ and can be used to construct an approximation of vector $\mathbb{U}$ at order $\left(n_{g}, n_{\ell}\right)$ such that

$$
\mathbb{U}^{\left(n_{g}, n_{\ell}\right)}=\sum_{\alpha=1}^{n_{g}} q_{\alpha}^{g} \boldsymbol{\phi}_{\alpha}^{g}+\sum_{\beta=1}^{n_{\ell}} q_{\beta}^{\ell} \boldsymbol{\phi}_{\beta}^{\ell}
$$

where $q_{1}^{g}, \ldots, q_{n_{g}}^{g}$ are the global coordinated $q_{1}^{\ell}, \ldots, q_{n_{\ell}}^{\ell}$ are the local coordinates. The latter equation can be rewritten as

$$
\mathbb{U}^{\left(n_{g}, n_{\ell}\right)}=\left[\Phi^{g}\right] \mathbf{q}^{g}+\left[\Phi^{\ell}\right] \mathbf{q}^{\ell}
$$

where $\left[\Phi^{g}\right]=\left[\boldsymbol{\phi}_{1}^{g}, \ldots, \boldsymbol{\phi}_{n_{g}}^{g}\right],\left[\Phi^{\ell}\right]=\left[\boldsymbol{\phi}_{1}^{\ell}, \ldots, \boldsymbol{\phi}_{n_{\ell}}^{\ell}\right], \mathbf{q}^{g}=\left(q_{1}^{g}, \ldots, q_{n_{g}}^{g}\right)$ and $\mathbf{q}^{\ell}=$ 
$\left(q_{1}^{\ell}, \ldots, q_{n_{\ell}}^{\ell}\right)$

\subsection{Reduced-order matrix equation.}

The reduced-order model is constructed by projecting matrix equation (32) using the global basis and the local basis. Let $n_{t}=n_{g}+n_{\ell}$ and let $\mathbf{q}(\omega)=\left(\mathbf{q}^{g}(\omega), \mathbf{q}^{\ell}(\omega)\right)$ be the vector of the $n_{t}$ generalized coordinates. Then, for all $\omega$ in $B$, vector $\mathbf{q}(\omega)$ is solution the following reduced matrix equation

$$
\left(-\omega^{2}[M]+i \omega[D]+[K]\right) \mathbf{q}(\omega)=\tilde{\mathbf{f}}(\omega),
$$

where $[M],[D]$ and $[K]$ are the $\left(n_{t} \times n_{t}\right)$ positive-definite mass, damping and stiffness generalized matrices defined by block as follows

$[M]=\left[\begin{array}{cc}M^{g g} & M^{g \ell} \\ \left(M^{g \ell}\right)^{T} & M^{\ell \ell}\end{array}\right],[D]=\left[\begin{array}{cc}D^{g g} & D^{g \ell} \\ \left(D^{g \ell}\right)^{T} & D^{\ell \ell}\end{array}\right],[K]=\left[\begin{array}{cc}K^{g g} & K^{g \ell} \\ \left(K^{g \ell}\right)^{T} & K^{\ell \ell}\end{array}\right]$.

Let $A$ (or $\mathbb{A}$ ) denote $M, D$ or $K$ (or $\mathbb{M}, \mathbb{D}$ or $\mathbb{K}$ ). Then the global, local and coupling blocks are respectively defined by

$$
\left[A^{g g}\right]_{\alpha \beta}=\left(\phi_{\alpha}^{g}\right)^{T}[\mathbb{A}] \phi_{\beta}^{g},\left[A^{\ell \ell}\right]_{\alpha \beta}=\left(\phi_{\alpha}^{\ell}\right)^{T}[\mathbb{A}] \phi_{\beta}^{\ell},\left[A^{g \ell}\right]_{\alpha \beta}=\left(\phi_{\alpha}^{g}\right)^{T}[\mathbb{A}] \boldsymbol{\phi}_{\beta}^{\ell} .
$$

The blocks $\left[K^{g g}\right]$ and $\left[K^{\ell \ell}\right]$ are diagonal by construction. The vector of the generalized forces is written as $\tilde{\mathbf{f}}(\omega)=\left(\tilde{\mathbf{f}}^{g}(\omega), \tilde{\mathbf{f}}^{\ell}(\omega)\right)$ where $\tilde{\mathbf{f}}_{\alpha}^{g}(\omega)=$ $\left(\phi_{\alpha}^{g}\right)^{T} \mathbb{F}(\omega)$ and $\tilde{\mathbf{f}}_{\alpha}^{\ell}(\omega)=\left(\phi_{\alpha}^{\ell}\right)^{T} \mathbb{F}(\omega)$.

\section{Stochastic reduced-order computational model.}

Several types of uncertainties may exist for a computational model representing a family of nominally identical dynamical structures:

- The parameters uncertainties are induced by (1) the variability of some parameters of the structure that may exist between two specimens of the structure (induced by the manufacturing tolerances, damage or transformation occurring during the life cycle, ...), (2) by the lack of knowledge related to these parameters (such epistemic uncertainties may be reduced if more experimental data are available) and (3) by a natural randomness that may exist inside one specimen of the structure (random forces, random configurations during the cycle of life, ...).

- The model uncertainties are due to the modelling choices that have been done during the construction of the computational model: constitutive laws, 
geometry details, boundary conditions, and so on.

- The experimental uncertainties are concerned if the computational model is updated using experimental data. In this case, the experimental data may be uncertain due to the measurement errors, the choice of the experimental configuration, and so on.

The present paper is only focused of parameters uncertainties and model uncertainties. In a complex system such as an automotive vehicle, there exit a large amount of possible sources of both parameters uncertainties and model uncertainties. It is then very difficult to try to model separately each source of uncertainty. In the present paper, all the sources of uncertainties are taken into account by constructing a probabilistic model of uncertainties at the reduced-order model level directly by using a non-parametric probabilistic approach for which the matrices of the reduced-order model are replaced by random matrices (see [30]). One of the advantages of this method is that the probabilistic model of all the sources of uncertainties are controlled by a small numbers of dispersion parameters. This feature facilitates an experimental identification (if experimental data are available) or a sensitivity analysis.

The objective here is to take the advantages of the global/local separation that has been introduced previously in order to control separately the level of fluctuations for the global displacements and the level of fluctuations for the local displacements. In [32], a global/local probabilistic model was proposed in which only the global and local blocks of the generalized matrices are replaced by random matrices while the coupling blocks are kept deterministic. In the approach that is proposed below the probabilistic modeling is improved by adding a probabilistic modeling of the coupling blocks. The proposed construction ensures the positive-definiteness of the random generalized matrices.

The blocks of the generalized matrices in Eq. (41) can be rewritten as

$$
\left[A^{g g}\right]=\left[P^{g}\right]^{T}[A]\left[P^{g}\right], \quad\left[A^{g \ell}\right]=\left[P^{g}\right]^{T}[A]\left[P^{\ell}\right], \quad\left[A^{\ell \ell}\right]=\left[P^{\ell}\right]^{T}[A]\left[P^{\ell}\right],
$$

where $\left[P^{g}\right]$ and $\left[P^{\ell}\right]$ are projection matrices on the global coordinates and local coordinates respectively and are then such that $\left[P^{g}\right]=\left[I_{n_{g}} 0_{n_{\ell}}\right]$ et $\left[P^{\ell}\right]=$ $\left[0_{n_{g}} I_{n_{\ell}}\right]$. The probabilistic model of uncertainties is constructed using a nonparametric probabilistic approach in which the matrix $[A]$ is replaced by (1) the random matrix $\left[\mathbf{A}^{g}\right]$ for the global contributions and (2) the matrix $\left[\mathbf{A}^{\ell}\right]$ for the local contributions (see Appendix B for the generation of independent 
realizations of positive-definite random matrices). The Cholesky factorisation of these matrices yields

$$
\left[\mathbf{A}^{g}\right]=\left[\mathbf{L}_{A}^{g}\right]^{T}\left[\mathbf{L}_{A}^{g}\right], \quad\left[\mathbf{A}^{\ell}\right]=\left[\mathbf{L}_{A}^{\ell}\right]^{T}\left[\mathbf{L}_{A}^{\ell}\right] .
$$

The deterministic blocks in Eq. (41) are then replaced by the following random blocks

$$
\left[\mathbf{A}^{g g}\right]=\left[P^{g}\right]^{T}\left[\mathbf{A}^{g}\right]\left[P^{g}\right], \quad\left[\mathbf{A}^{g l}\right]=\left[P^{g}\right]^{T}\left[\mathbf{L}_{A}^{g}\right]^{T}\left[\mathbf{L}_{A}^{\ell}\right]\left[P^{\ell}\right], \quad\left[\mathbf{A}^{\ell \ell}\right]=\left[P^{\ell}\right]^{T}\left[\mathbf{A}^{\ell}\right]\left[P^{\ell}\right],
$$

The randomness of the random coupling blocks $\left[\mathbf{A}^{g \ell}\right]$ is then due to both the global randomness and the local randomness. Then, the random matrix $[\mathbf{A}]$ is written as

$$
[\mathbf{A}]=\left[\begin{array}{cc}
\mathbf{A}^{g g} & \mathbf{A}^{g \ell} \\
\left(\mathbf{A}^{g \ell}\right)^{T} & \mathbf{A}^{\ell \ell}
\end{array}\right]=\left[\mathbf{L}_{A}^{g} P^{g} \mathbf{L}_{A}^{\ell} P^{\ell}\right]^{T}\left[\mathbf{L}_{A}^{g} P^{g} \mathbf{L}_{A}^{\ell} P^{\ell}\right]
$$

which shows that random matrix $[\mathbf{A}]$ is positive definite almost surely. The probabilistic model of uncertainties for random matrix $[\mathbf{A}]$ is controlled by two dispersion parameters $\delta_{A, g}$ and $\delta_{A, \ell}$ which are associated with the random matrices $\left[\mathbf{A}^{g}\right]$ and $\left[\mathbf{A}^{\ell}\right]$ respectively. Then considering the three random matrices $[\mathbf{M}],[\mathbf{D}]$ and $[\mathbf{K}]$, the complete probabilistic model of uncertainties is controlled by six dispersion parameters $\delta_{M, g}, \delta_{M, \ell}, \delta_{D, g}, \delta_{D, \ell}, \delta_{K, g}$ and $\delta_{K, \ell}$. These six parameters allows to control the level of fluctuations of the global displacements and the level of fluctuations of the local displacements separately for each random operator. Concerning the values of these parameters two situations may exist:

- If experimental data are available then the dispersion parameters can be identified by solving an inverse stochastic problem (maximum likelihood method for instance). Since the dispersion parameters are related to a nonparametric model of all the sources of uncertainties, the identified values can be used for other computational models related to structures which are similar to the structure that has been used to identify the dispersion parameters. Indeed such an identification is very expensive and can not be performed for each designed structure.

- If no experimental data is available then a sensitivity analysis with respect to uncertainties can be performed by analysing the response of the stochastic computational model for several sets of values for the dispersion parameters. 


\section{Application.}

In this section the methodology is validated through a numerical application which is relatively simple but presents two structural scales which allow the presence of both (1) global displacements and (2) non-negligible local displacements even for low frequencies. The nominal and stochastic computational models are constructed. For this application, the stochastic model is used to perform a sensitivity analysis with respect to the global and the local uncertainties. For a real structure for which experimental data would be available, the value of the dispersion parameters could be identified and the uncertainty on the outputs could be quantified.

\subsection{Nominal computational model.}

The mesh of the FE model is represented on Figure 1. This structure is

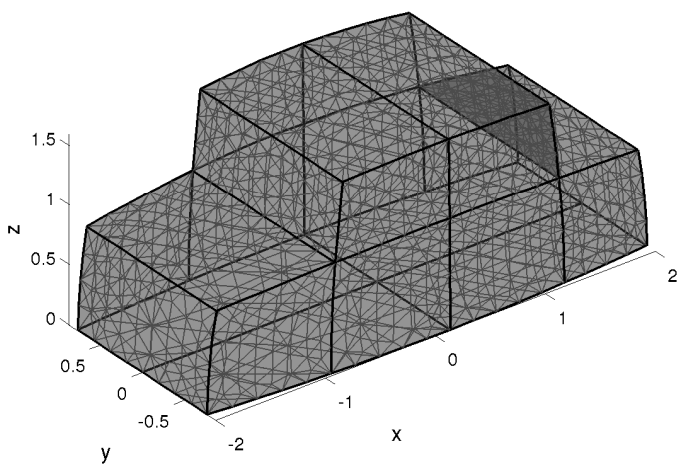

Figure 1: FE mesh of the nominal computational model.

made up of and (1) Euler beams (thick black lines on Fig. 1) which constitute the master stiff part and (2) Kirchhof plates which constitutes flexible substructures. The plates are curved (but the elements are flat) and have different deterministic prescribed values for the Young's modulus within the range $[6.01,6.74] \times 10^{10} \mathrm{~Pa}$. The bottom-front line and the bottom-rear line are fixed (see Fig. 2). The frequency band of analysis is $B=2 \pi \times] 0,200] \mathrm{rad} / \mathrm{s}$. The structure has $m=5,844 \mathrm{DOF}$. In the frequency band ]0,247] $\mathrm{Hz}$, the nominal computational model has 250 classical elastic modes. 


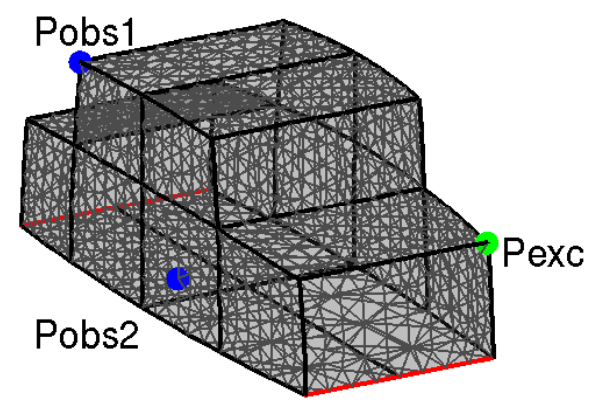

Figure 2: Fixed parts (red line), excitation point (green) and observation points (bleu).

\subsection{Reduced-order computational model.}

The global basis and the local basis are constructed using the methodology introduced in Sections 3 and 4. Three types of kinematic reduction corresponding to the three particular cases developed in Section 3.2.2 are analysed. Each one is based on a decomposition of the domain into 30 subdomains (see Fig. 3). These subdomains are constructed using the Fast Marching method (see $[15,26,1])$ which allows to propagate fronts from a set of initial points. The Kinematic 1 corresponds to a uniform displacement in each subdomain (first particular case in Section 3.2.2), the Kinematic 2 corresponds to a rigid body displacement in each subdomain (second particular case in Section 3.2.2) and the Kinematic 3 corresponds to a linear displacement in each subdomain (third particular case in Section 3.2.2). For Kinematic 1, the structure exhibits 30 global eigenvectors and 235 local eigenvectors in the frequency band $] 0,247] \mathrm{Hz}$. For Kinematic 2, the structure exhibits 46 global eigenvectors and 223 local eigenvectors in the frequency band ]0, 247] Hz. For Kinematic 3, the structure exhibits 59 global eigenvectors and 214 local eigenvectors in the frequency band ]0, 247] Hz. Since global 


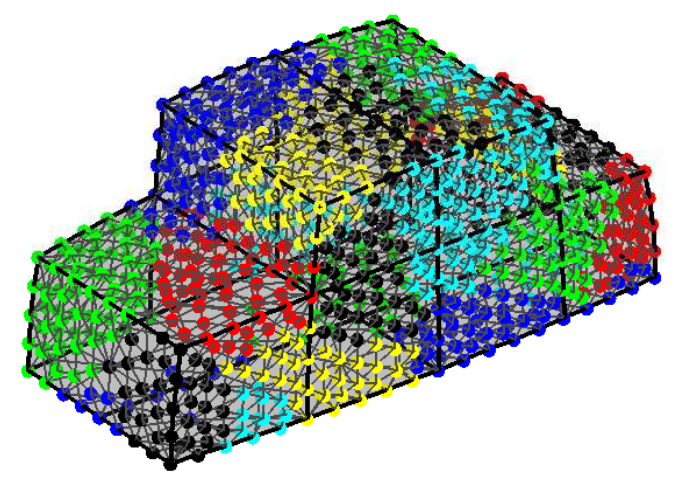

Figure 3: Decomposition of the domain into 30 subdomains. Each color represents a subdomain.

eigenvectors and local global eigenvectors are not classical elastic modes, the total number of global eigenvectors and local eigenvectors in $] 0,247] \mathrm{Hz}$ is not equal to number of classical elastic modes in ]0,247] Hz. For Kinematic 2, the histogram of the global eigenfrequencies and the histogram of the local eigenfrequencies are plotted on Fig. 4. It can be seen that in the frequency band $] 0,247] \mathrm{Hz}$ the global eigenvectors and local eigenvectors overlap completely. For Kinematic 2, global eigenvector $\phi_{1}^{g}$ and $\phi_{16}^{g}$ are plotted on Fig. 5 (these two eigenvectors are flexural modes of structure around $y$-direction and $z$-direction respectively) and local eigenvector $\phi_{1}^{l}$ and $\phi_{64}^{l}$ are plotted on Fig 6 (these two local eigenvectors are localized on the front plate and on the roof respectively). A force equal to $1 \mathrm{~N}$ is applied at an excitation point Pexc (see Fig. 2) along $y$-direction. A Rayleigh damping model with damping rate 0.04 at frequencies $30 \mathrm{~Hz}$ and $200 \mathrm{~Hz}$ is used to construct the damping matrix. The frequency response is calculated along $y$-direction at two observation points: (1) Pobs $_{1}$ located on the stiff master part of the structure and (2) $\mathrm{Pobs}_{2}$ located on a flexible panel (see Fig. 2). For Kinematic 1, Kinematic 2 and Kinematic 3, the frequency response is calculated 


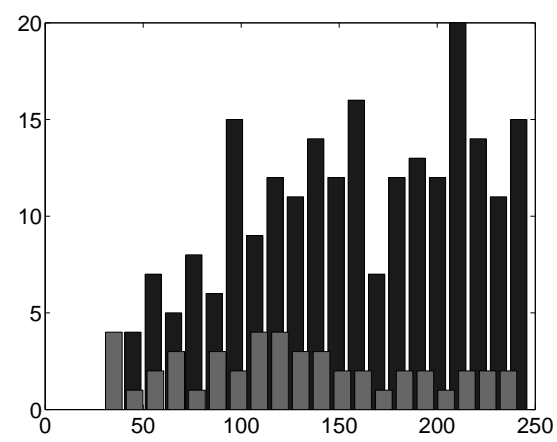

Figure 4: Modal density for the global eigenfrequencies (grey) and the local eigenfrequencies (black).
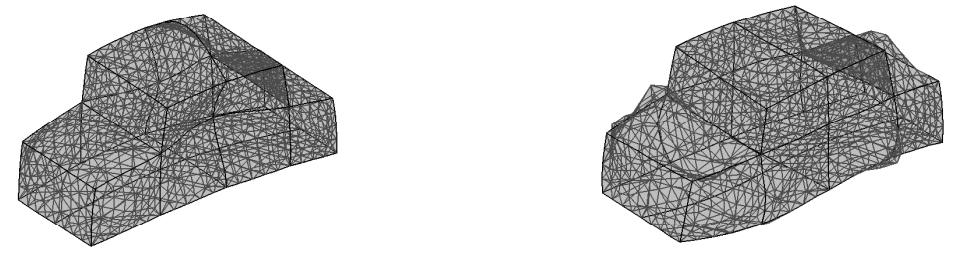

Figure 5: Global eigenvectors $\phi_{1}^{g}$ and $\phi_{16}^{g}$.
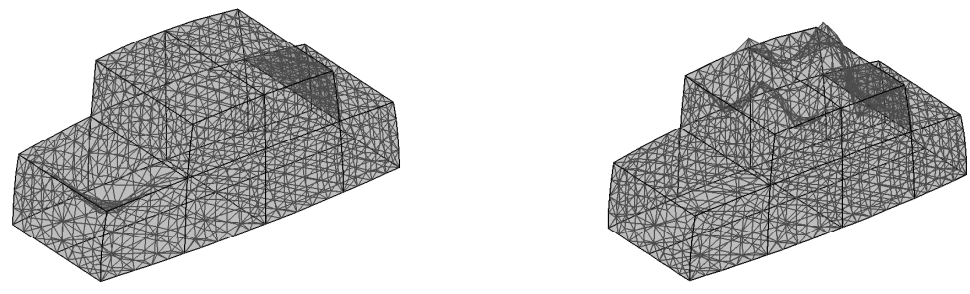

Figure 6: Local eigenvectors $\phi_{1}^{l}$ and $\phi_{64}^{l}$. 
using different projection bases: Global eigenvectors only $\left(n_{g}=30\right.$ for Kinematic $1, n_{g}=46$ for Kinematic $2, n_{g}=59$ for Kinematic 3 and $n_{\ell}=0$ ), local eigenvectors only $\left(n_{g}=0, n_{\ell}=235\right.$ for Kinematic $1, n_{\ell}=223$ for Kinematic 2 and $n_{\ell}=214$ for Kinematic 3 ) and both global and local eigenvectors $\left(n_{g}=30\right.$ for Kinematic 1, $n_{g}=46$ for Kinematic $2, n_{g}=59$ for Kinematic 3, $n_{\ell}=235$ for Kinematic $1, n_{\ell}=223$ for Kinematic 2 and $n_{\ell}=214$ for Kinematic 3). The responses for Kinematic 1 are plotted on Fig. 7, the responses for Kinematic 2 are plotted on Fig. 9 and the responses for Kinematic 3 are plotted on Fig. 10. For point Pobs $_{1}$, the three first resonances correspond
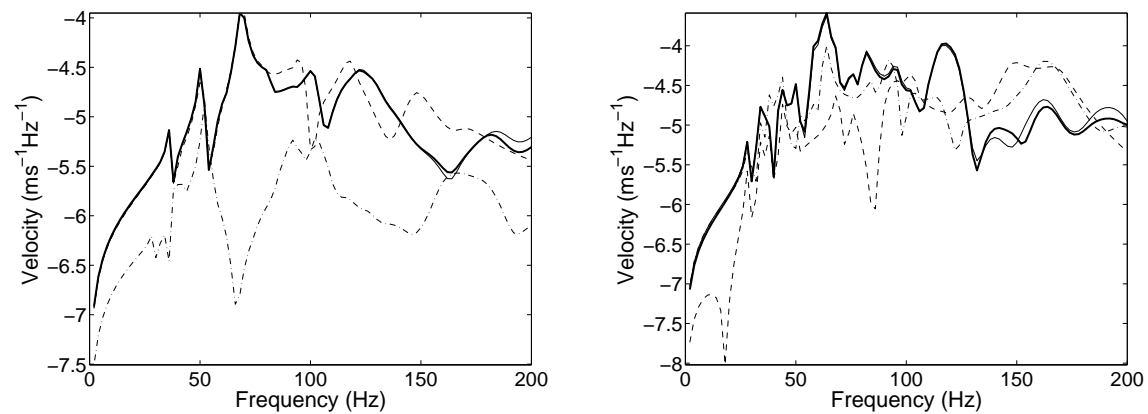

Figure 7: Kinematic 1: Frequency response at points $P_{0 b s_{1}}$ (left figure) and Pobs 2 (right figure). Exact response (thick solid line), response with global eigenvector (dashed line), response with local eigenvectors (mixed line) and response with both global and local eigenvectors (thin solid line, superimposed with exact response almost everywhere).

to global elastic modes. It can be seen on figures 7 and 9 that:

- For point Pobs 1 located in the master stiff part of the structure, the contributions of the local displacements are small while for point $\mathrm{Pobs}_{2}$ located in the flexible part, the contributions of the local displacements are larger.

- The union of the global eigenvectors and the local eigenvectors allows to compute responses which are very closed to the exact responses. The small differences are due to truncation effects of the global basis and the local basis. - The Kinematic 2 which corresponds to a finer kinematic reduction for the kinetic energy than Kinematic 1 allows a better global/local separation to be performed. Indeed, it can be seen that for the Kinematic 1, in the low frequency range, the local eigenvectors tend to synthesise resonances corresponding to global displacements. That is not the case for Kinematic 2. The Kinematic 3 which corresponds to a finer kinematic reduction than Kinematic 2 allows to improve a little more the global/local separation. 

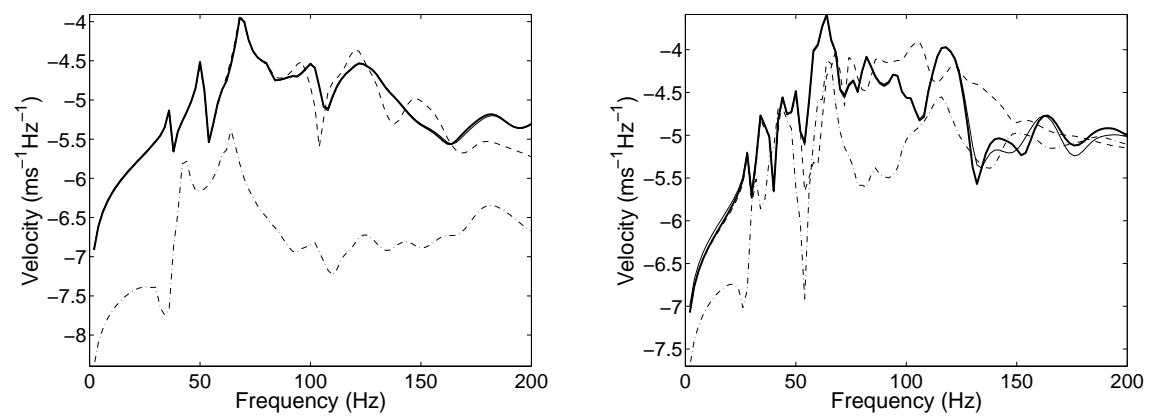

Figure 8: Kinematic 2: Frequency response at points $\mathrm{Pobs}_{1}$ (left figure) and $\mathrm{Pobs}_{2}$ (right figure). Exact response (thick solid line), response with global eigenvector (dashed line), response with local eigenvectors (mixed line) and response with both global and local eigenvectors (thin solid line, superimposed with exact response almost everywhere).
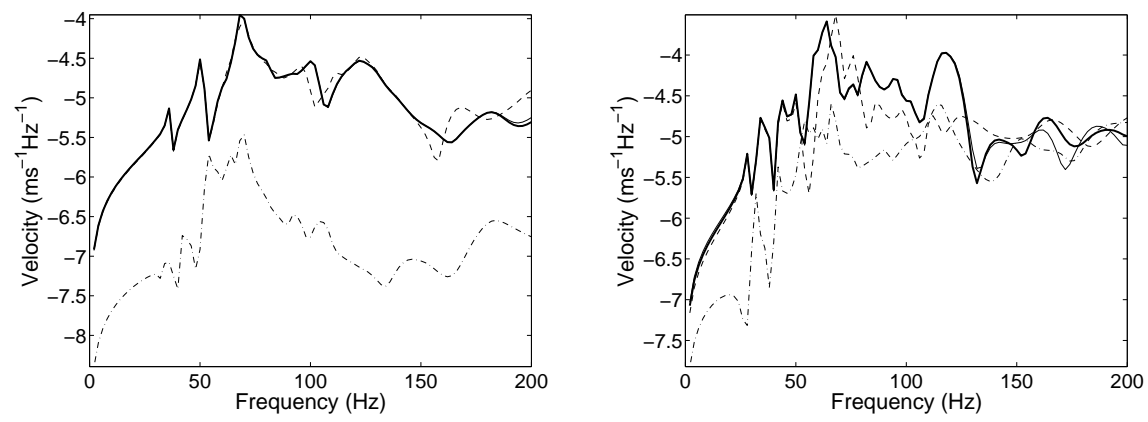

Figure 9: Kinematic 3: Frequency response at points $\mathrm{Pobs}_{1}$ (left figure) and Pobs 2 (right figure). Exact response (thick solid line), response with global eigenvector (dashed line), response with local eigenvectors (mixed line) and response with both global and local eigenvectors (thin solid line, superimposed with exact response almost everywhere).

\subsection{Stochastic reduced-order computational model.}

The Stochastic reduced-order computational model is constructed for Kinematic 2 only. The random response is analysed using the Monte Carlo simulation method with $n_{s}=1000$ realizations, for three cases: (1) For the first one, only the global blocks are random with $\delta_{M, g}=\delta_{D, g}=\delta_{K, g}=0.2$ and $\delta_{M, \ell}=\delta_{D, \ell}=\delta_{K, \ell}=0$, (2) for the second one, only the local blocks are random with $\delta_{M, g}=\delta_{D, g}=\delta_{K, g}=0$ and $\delta_{M, \ell}=\delta_{D, \ell}=\delta_{K, \ell}=0.2$ and (3) the third one, both the global and local blocks are random with $\delta_{M, g}=\delta_{D, g}=\delta_{K, g}=0.2$ and $\delta_{M, \ell}=\delta_{D, \ell}=\delta_{K, \ell}=0.2$. The random response 
for these three cases are plotted on figures 10-12. It can be seen on Fig. 10
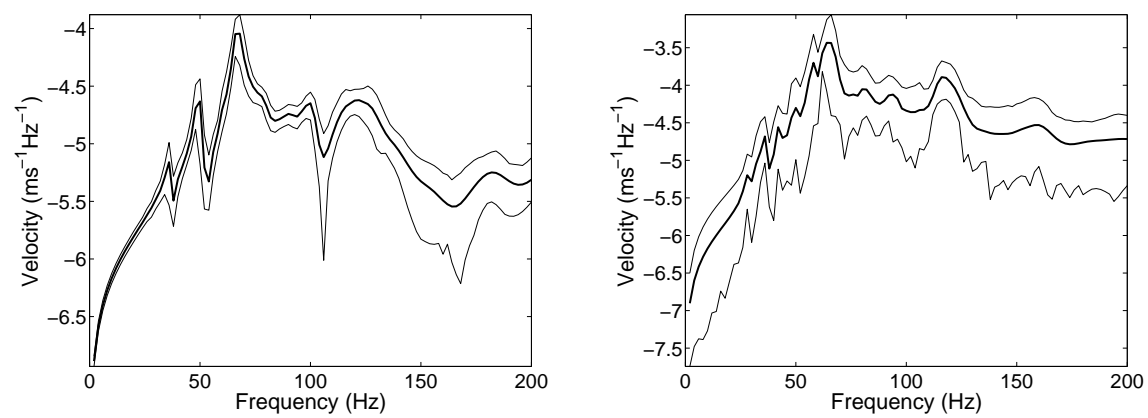

Figure 10: Random global blocks, deterministic local blocks: Random frequency response at points $P_{o b s}$ (left figure) and $P_{o b s}$ (right figure). Mean response (thick solid line) and confidence region with probability level 0.95 (thin solid lines).
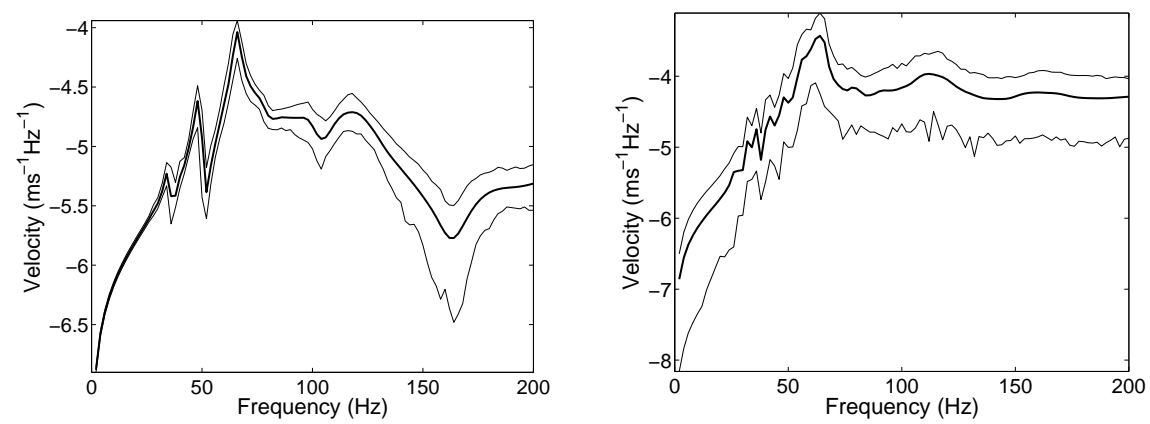

Figure 11: Deterministic global blocks, random local blocks: Random frequency response at points $P_{o b s}$ (left figure) and Pobs 2 (right figure). Mean response (thick solid line) and confidence region with probability level 0.95 (thin solid lines).

that the randomness on the global blocks yields fluctuations of the random response in all the frequency band $B$. Indeed the global eigenfrequencies are regularly spaced in all the frequency band $B$. Figure 11 shows that for point $P_{o b s}$ located on the stiff part, the randomness on the local blocks yields fluctuations of the random response mainly in the frequency band $] 75,200] \mathrm{Hz}$ where the local contributions are more important. For point $\mathrm{Pobs}_{2}$ located on the flexible part, the randomness on the local blocks yields large fluctuations in all the frequency band $] 0,200] \mathrm{Hz}$ with a smoothing of the mean 

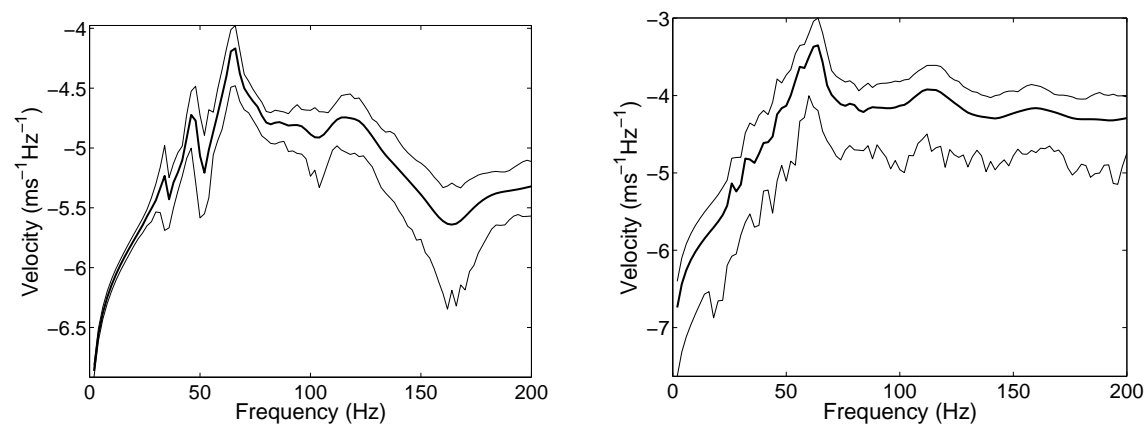

Figure 12: Random global blocks, random local blocks: Random frequency response at points $P o b s_{1}$ (left figure) and $P_{o b s}$ (right figure). Mean response (thick solid line) and confidence region with probability level 0.95 (thin solid lines).

response for large frequencies. In Fig. 12, the global randomness and the local randomness are cumulated yielding fluctuations in all the frequency band ]0 , 200] $\mathrm{Hz}$ with larger fluctuations in the frequency band $] 75,200] \mathrm{Hz}$ where the local contributions are more important.

\section{Conclusions.}

A new methodology for the construction of a probabilistic reduced-order model adapted to the low- and mid-frequency structural dynamics has been presented. The projection operators which are needed for the construction of the global eigenvalue problem and the local eigenvalue problem are constructed by minimizing the kinetic energy of the error induced by the kinematic reduction. Several classes of kinematic reduction can be constructed. The application shows that the rigid body kinematic reduction allows a good global/local separation to be performed. Then a probabilistic model of uncertainties can be implemented using a non-parametric approach for the global matrix blocks and the local matrix blocks separately allowing to control the global fluctuations and the local fluctuations separately. This possibility of controlling these two types of fluctuation is of most importance since, in general, the local displacements are more sensitive to uncertainties than the global displacements.

\section{Acknowledgements}


This research was supported by the "Agence Nationale de la Recherche", Contract HiMoDe, ANR-12-JS09-0014.

\section{Appendix A. Independence of the global basis and the local basis.}

It is shown here that the global basis $\left\{\boldsymbol{\phi}_{1}^{g}, \ldots, \boldsymbol{\phi}_{n_{r}}^{g}\right\}$ is linearly independent of the local basis $\left\{\phi_{1}^{\ell}, \ldots, \phi_{n_{c}}^{\ell}\right\}$.

Let $\boldsymbol{u}=a \boldsymbol{\phi}_{\alpha}^{g}+b \boldsymbol{\phi}_{\beta}^{\ell}$ with $(a, b) \in \mathbb{R}^{2}, \alpha \in\left\{1, \ldots, n_{r}\right\}$ and $\beta \in\left\{1, \ldots, n_{c}\right\}$. We then have to show that $\boldsymbol{u}=\mathbf{0}$ implies that $a=0$ and $b=0$. We have $\boldsymbol{u}=\mathbf{0}$ implies that $a[\mathbb{K}] \boldsymbol{\phi}_{\alpha}^{g}+b[\mathbb{K}] \boldsymbol{\phi}_{\beta}^{\ell}=0$ which implies that $a \lambda_{\alpha}^{g}\left[\mathbb{M}^{r}\right] \boldsymbol{\phi}_{\alpha}^{g}+$ $b \lambda_{\beta}^{l}\left[\mathbb{M}^{c}\right] \phi_{\beta}^{\ell}=0$. Then using Eqs. (33) and (34), this implies that $[\mathbb{M}]\left(a \lambda_{\alpha}^{g}\left[H^{r}\right] \phi_{\alpha}^{g}+\right.$ $\left.b \lambda_{\beta}^{l}\left[H^{c}\right] \phi_{\beta}^{\ell}\right)=0$ and then since matrix $[M]$ is positive-definite $a \lambda_{\alpha}^{g}\left[H^{r}\right] \phi_{\alpha}^{g}+$ $b \lambda_{\beta}^{l}\left[H^{c}\right] \phi_{\beta}^{\ell}=0$. The term $a \lambda_{\alpha}^{g}\left[H^{r}\right] \phi_{\alpha}^{g}$ belongs to the subspace $\mathcal{Y}^{r}$ and the term $b \lambda_{\beta}^{l}\left[H^{c}\right] \phi_{\beta}^{\ell}$ belongs to the subspace $\mathcal{Y}^{c}$. Since we have $\mathbb{R}^{m}=\mathcal{Y}^{r} \oplus \mathcal{Y}^{c}$, this implies that these two terms are both zero. Finally, the global eigenfrequencies and the local eigenfrequecies being strictly positive, it can be concluded that $a=0$ and $b=0$.

\section{Appendix B. Generator of independent realizations for normalized symmetric positive-definite random matrices.}

In this appendix, we recall the formulation [30] for the generator of independent realizations of symmetric positive-definite random matrices. Let $[\mathbf{A}]$ be $\left[\mathbf{A}^{g}\right]$ or $\left[\mathbf{A}^{\ell}\right]$ introduced in Section 5 and for which the mean value is denoted $[A]$. For more convenience, the random matrix $[\mathbf{A}]$ is normalized as follow. Matrix $[A]$ being positive definite, its Cholesky decomposition yields

$$
[A]=\left[\underline{L}_{A}\right]^{T}\left[\underline{L}_{A}\right],
$$

in which $\left[\underline{L}_{A}\right]$ is an upper triangular matrix in the set $\mathbb{M}_{n_{t}}(\mathbb{R})$ of all the $\left(n_{t} \times n_{t}\right)$ real matrices. Then, the random matrix $[\mathbf{A}]$ can be rewritten as

$$
[\mathbf{A}]=\left[\underline{L}_{A}\right]^{T}[\mathbf{G}]\left[\underline{L}_{A}\right],
$$

The random matrix $[\mathbf{G}]$ is written as $[\mathbf{G}]=[\mathbf{L}]^{T}[\mathbf{L}]$, in which $[\mathbf{L}]$ is an upper triangular random matrix with values in $\mathbb{M}_{n_{t}}(\mathbb{R})$ such that:

(1) random variables $\left\{[\mathbf{L}]_{j j^{\prime}}, j \leq j^{\prime}\right\}$ are independent; 
(2) for $j \leq j^{\prime}$, real-valued random variables $[\mathbf{L}]_{j j^{\prime}}$ can be written as $[\mathbf{L}]_{j j^{\prime}}=$ $\sigma_{n_{t}} U_{j j^{\prime}}$ in which $\sigma_{n_{t}}=\delta_{A}\left(n_{t}+1\right)^{-1 / 2}$ and where $U_{j j^{\prime}}$ is a real-valued Gaussian random variable with zero mean and variance equal to 1 ;

(3) for $j=j^{\prime}$, positive-valued random variables $[\mathbf{L}]_{j j}$ can be written as $[\mathbf{L}]_{j j}=\sigma_{n_{t}} \sqrt{2 V_{j}}$ in which $\sigma_{n_{t}}=\delta_{A}\left(n_{t}+1\right)^{-1 / 2}$ and where $V_{j}$ is a positivevalued gamma random variable whose probabilty density function $p_{V_{j}}(v)$ with respect to $d v$ is written as

$$
p_{V_{j}}(v)=1_{\mathbb{R}^{+}}(v) \frac{1}{\Gamma\left(\frac{n_{t}+1}{2 \delta_{F}^{2}}+\frac{1-j}{2}\right)} v^{\frac{n_{t}+1}{2 \delta_{A}^{2}-\frac{1+j}{2}}} e^{-v},
$$

in which $\delta_{A}$ is the dispersion parameter defined by

$$
\delta_{A}=\left\{\frac{1}{n_{t}} E\left\{\left\|[\mathbf{G}]-\left[I_{n_{t}}\right]\right\|\right\}_{F}^{2}\right\}^{1 / 2},
$$

and which has to be chosen such that

$$
0<\delta_{A}<\sqrt{\left(n_{t}+1\right)\left(n_{t}+5\right)^{-1}} .
$$

[1] A. Arnoux, A. Batou, C. Soize, L. Gagliardini, Stochastic reduced order computational model of structures having numerous local elastic modes in low frequency dynamics, Journal of Sound and Vibration, 332, 3667$3680(2013)$

[2] J.P. Aubin, Applied Functional Analysis, 2nd Edition, Wiley (2000)

[3] A. Barbarulo, H. Riou, L. Kovalevsky, P. Ladeveze, PGD-VTCR: A Reduced Order Model Technique to Solve Medium Frequency Broad Band Problems on Complex Acoustical Systems, Strojniski Vestnik Journal of Mechanical Engineering, 60(5), 307-313 (2014)

[4] A. Batou, C. Soize, Stochastic reduced-order model in low-frequency dynamics in presence of numerous local elastic modes, Proceedings of RASD 2010 - 10th International Conference on Recent Advances in Structural Dynamics, Southampton, 12-14 July (2010).

[5] M.A. Ben Souf, M.N. Ichchou, N. Bareille, M. Haddar, On the dynamics of uncertain coupled structures through a wave based method in mid- 
and high-frequency ranges, Computational Mechanics, 52(4), 849-860 (2013)

[6] G. Borello L. Gagliardini, L. Houillon, L. Petrinelli, Virtual sea-feabased modeling of mid-frequency structure-borne noise, Sound and Vibration, 39(1), 22-28 (2005)

[7] M. Chevreuil, P. Ladevèze, P. Rouch, Transient analysis including the low- and the medium-frequency ranges of engineering structures, Computers and Structures, 85(17-18), 1431-1444 (2007)

[8] V. Cotoni, P.J. Shorter, R.S. Langley. Numerical and experimental validation of the hybrid finite element-statistical energy analysis method, Journal of the Acoustical Society of America, 259-270 (2007)

[9] W. Desmet, P. Sas, D. Vandepitte, A comparison between an indirect trefftz method and the finite-element method for solving coupled vibroacoustic problems, Journal of Acoustic Society of America, 106(4), 2118 (1999).

[10] J.F. Durand, C. Soize, L. Gagliardini, Structural-acoustic modeling of automotive vehicles in presence of uncertainties and experimental identification and validation, J. Acoust. Soc. Am., 124(3), 1513-1525 (2008)

[11] B. Fraeijs De Veubeke, The dynamics of flexible bodies , International Journal of Engineering Science, 14(10), 895-913 (1976)

[12] C. Grandmont, Y. Maday, P. Mtier, Modeling and Analysis of an Elastic Problem with Large Displacements and Small Strains, Journal of Elasticity, 87(1), 29-72 (2007)

[13] R.J. Guyan, Reduction of Stiffness and Mass Matrices, AIAA Journal, 3, 380-388 (1965)

[14] M. Kassem, C. Soize, L. Gagliardini, Structural partitioning of complex structures in the medium-frequency range. An application to an automotive vehicle, Journal of Sound and Vibration, 330(5), 937-946 (2011)

[15] R. Kimmel, J.A. Sethian, Computing geodesic paths on manifolds, Proc. Natl. Acad. Sci. USA, 8431-8435 (1998) 
[16] M. Kompella, R.J. Berhard, Measurement of the statistical variation of structural-acoustic characteristics of automative vehicules, SAE Noise and Vibration Conference (1993)

[17] L. Kovalevsky, H. Riou, P. Ladeveze, A Trefftz approach for mediumfrequency vibrations of orthotropic structures, Computers \& Structures, $143,85-90(2014)$

[18] P. Ladevze, A new computational approach for structure vibrations in the medium frequency range, Comptes Rendus Acadmie des Sciences Paris, 332(2b), 849-856 (1996)

[19] R.S. Langley, V. Cotoni, Response variance prediction for uncertain vibro-acoustic system using a hybrid deterministic-statistical method, Journal of the Acoustical Society of America, 3445-3463 (2007)

[20] R.S. Langley, J.A. Cordioli, Hybrid deterministic-statistical analysis of vibroacoustic system domain couplings on statistical components, Journal of Sound and Vibration, 893-912 (2009)

[21] C. Lecomte, A frequency averaging framework for the solution of complex dynamic systems, Proceedings of the Royal Society A-Mathematical Physical and Engineering Sciences, 470(2166), 20130743 (2014)

[22] G. Mueller, M. Buchschmid, Hybrid approaches for vibroacoustical problems based on the finite element method and statistical energy analysis, Wave Motion, 51(4), 622-634 (2014)

[23] R. Ohayon, C. Soize, Structural Acoustics and Vibration. Academic Press, San Diego (1998)

[24] E. Reynders, R.S. Langley, A. Dijckmans, G. Vermeir, A hybrid finite element - statistical energy analysis approach to robust sound transmission modeling, Journal of Sound and Vibration, 333(19), 4621-4636 (2014)

[25] P. Rouch, C. Blanzé, Vibrational analysis of structures with stochastic interfaces in the medium-frequency range: Experimental validation on a touch screen, Journal of Sound and Vibration, 333 (6), 1612-1628 (2014) 
[26] J.A. Sethian, A. Vladimirsky, Fast methods for the Eikonal and related Hamilton-Jacobi equations on unstructured meshes, The Journal of Computational Physics, 148 (2-22) (1999).

[27] Soize, C., A Model and Numerical Method in the Medium Frequency Range for Vibroacoustic Predictions Using Theory of Structural fuzzy, J. Acoust. Soc. Am., 94(2), 849-866 (1993)

[28] P.J. Shorter, R.S. Langley, Vibro-acoustic analysis of complex systems, Journal of Sound and Vibration, 669-700 (2005)

[29] C. Soize, Reduced models in the medium frequency range for general dissipative structural-dynamics systems, European Journal of Mechanics A/Solids, 17(4), 657-685 (1998)

[30] C. Soize, Maximum entropy approach for modeling random uncertainties in transient elastodynamics, Journal of Acoustical Society of America,109(5), 1979-1996 (2001)

[31] C. Soize, Uncertain dynamical systems in the medium-frequency range, Journal of Engineering Mechanics-ASCE, 129(9), 1017-1027 (2003)

[32] C. Soize, A. Batou, Stochastic reduced-order model in low-frequency dynamics in presence of numerous local elastic modes, Journal of Applied Mechanics - Transactions of the ASME, 78(6), 061003 (2011)

[33] V.W. Sparrow, D.A. Russel, J.L. Rochat, Implementation of Discrete Fuzzy Structure Models in Mathematica, Int. J. Numer. Methods Eng., 37, 3005-3014 (1994)

[34] M. Strasberg, D. Feit, Vibration Damping of Large Structures Induced by Attached Small Resonant Structures, J. Acoust. Soc. Am., 99(1), 335-344 (1996)

[35] K. Vergote, C. Vanmaele, D. Vandepitte, W. Desmet, An efficient wave based approach for the time-harmonic vibration analysis of 3D plate assemblies, Journal of Sound and Vibration, 332(8), 1930-1946 (2013)

[36] Weaver, R.L., The Effect of an Undamped Finite Degree of Freedom Fuzzy Substructure: Numerical Solutions and Theoretical Discussion, J. Acoust. Soc. Am., 100(5), 3159-3164 (1996) 
[37] D. Zhu, H. Chen, X. Kong, Xiangjie, W.B. Zang, A hybrid finite element-energy finite element method for mid-frequency vibrations of built-up structures under multi-distributed loadings, Journal of Sound and Vibration, 333(22), 5723-5745 (2014) 